\title{
Tempus - Aspekt - Modus
}

Die lexikalischen und grammatischen Formen in den germanischen Sprachen

Herausgegeben von

Werner Abraham und Theo Janssen

Sonderdruck

Max Niemeyer Verlag Tübingen 1989 
Nominalreferenz, Zeitkonstitution, Aspekt, Aktionsart:

Eine semantische Erklärung ihrer Interaktion

Manfred Krifka (Universität Tübingen)

0. Einleitung und Uberblick

1. Nominalreferenz

2. Zeitkonstitution

3. Durative Adverbiale und Zeitspannen-Adverbiale

4. Progressiv und Partitiv

5. Perfektivität und Definitheit

6. Aspekt, Aktionsart, Zeitkonstitution

\section{o. Einleitung und trberblick}

Es ist mindestens seit Verkuyl (1972) bekannt, daß die nominalen Argumente von Verben Einfluß auf die Aspektklasse der Gesamtkonstruktion ausüben können. Nach dem gängigen Test für Telizität bzw. Atelizität - der Kombinierbarkeit mit durativen Adverbialen wie zehn Minuten lang und Zeitspannen-Adverbialen wie in zehn Minuten - ergebun sich Akzeptabilitätsunterschiede der folgenden Art:
(1) a. Anna aß zehn Minuten lang Äpfel/Apfelmus.
b. * Anna aß zehn Minuten lang einen Apfel.
(2) $\quad$ a. Anna aß in zehn Minuten Äpfel/Apfelmus
b. Anna a $B$ in zehn Minuten einen Apfel/ein Pfund Apfelmus.

In der vorliegenden Arbeit berichte ich über den Erklärungsansatz, den ich im Rahmen einer modelltheoretischen Semantik zur Beschreibung dieses Phänomens entwickelt habe. Ich konzentriere mich hierbei auf die zugrundeliegende Motivation und die intuitive Charakterisierung dieser Theorie. Leser, die an den Einzelheiten der Durchführung und an weiteren An wendungsmöglichkeiten der Theorie interessiert sind, seien auf Krifka (1987, 1989) verwiesen; ein forschungshistorischer Abriß zu alternativen Theorien findet sich in Krifka (1986). 
Darüber hinaus will ich zeigen. daß durch die begriffliche Präzisierung. die dieser Erklärungs ansatz erlaubt. sich eine fundierte tinterscheidung der Begriffe Zeitkonstitution. Aktionsant und Aspekt abzeichnet.

\section{Nominalreferenz}

Die nominale Distinktion, die für die Grundbeispiele (1.2) entscheidend ist, ist nicht, wie oftmals angenommen. die lexikalische Unterscheidung zwischen Individualnomina (count nouns) wie Apfel und Massennomina (mass nouns) wie tpfelmus. sondern eine andere, die beispiclsweise Ausdrücke wie ein Apfel, drei Apfel, zwei Cläser. Aplelmus, diese apfel oder auch Namen wie Anna auf der einen und Ausdrücke wie Apfel oder Apfelmus auf der anderen Seite zu Klassen zusammenfaßt.

Der wesentliche Unterschied zwischen diesen nominalen Ausdrücken wurde in Quine (1960) wie folgt bestimmt: Ausdrücke der zweiten Klasse haben die Eigenschaft der kumulativen Referenz: Wenn auf zwei Entitäten das Prädikat Apfel oder Apfelmus angewendet werden kann, dann kann dies auch auf die Zusammenfassung angewendet werden (kurz: Äpfel und Äpfel ergibt wieder Äpfel; Apfelmus und Apfelmus ergibt wieder Apfelmus). Dies ist bei Ausdrücken wie drei Apfel oder ein Pfund Apfelmus nicht der Fall: wenn dieses Prädikat auf zwei Entitäten angewendet werden kann. so ist es auf deren Zusammenfassung nicht mehr anwendbar (drei Apfel und drei Äpfel ergeben mehr als drei Äpfel: ein Pfund Apfelmus und ein Pfund Apfelmus ergibt mehr als ein Pfund Apfelmus).

Ausdrücke wie drei ̈̈pfel oder ein Pfund Apfelmus können einfach als nicht kunulativ beschrie ben werden. Darüber hinaus ist jedoch auch eine engere Charakterisierung möglich: Wenn ein Objekt unter das Prädikat drei Apfel (oder ein Pfund Apfelmus) fällt. so kann kein echter Teil davon unter dasselbe Prädikat fallen: ein echter Teil von drei Äpfeln sind eben keine drei Äpfel mehr, ein echter Teil eines pfundes Apfelmus kein Pfund Apfelınus. Ich nenne Prädikate. die diese Eigenschaft haben. Prädikate mit gequantelter Referenz. Als übergreifenden Begriff verwende ich Nominalreferenz.

In der l)efinition dieser Begriffe der kumulativen und der gequantelten Referenz spielt dic Operation der Zusammenfassung und die Teilbeziehung eine wesentliche Rolle. Das zugrundeliegende mathematische Modell eines Sumnenhalbverbandes wurde in Link (1983) auf die Semantik von Massennomina angewender. An dieser Stelle will ich die zugrundeliegende semantische $\mathrm{Bc}^{2}$ griffsbildung eher informell erläutern: eine svstematischere Behandlung findet sich in Krifka (1989). 
Zu je zwei Objekten X,y im Bereich der Entitäten, über die wir sprechen, gibt es deren Zusammenfassung oder Summe. notiert als xuv. Diese soll insbesondere kommurativ, idempotent und assoziativ sein: Kommutativ (d.h. $x u y=y u x)$, da es auf die Reihenfolge in der Zusammenfassung nicht ankommt: assoziativ (d.h. $x u(v u z)=(x u y) u z$ ), da es auf die Reihenfolge verschiedener $\mathrm{Zu}-$ sammenfassung nicht ankommt: idempotent (d.h. $x u X=X)$, da die Zusammenfassung einer Entität mit sich selbst diese nicht verändert.

Aut der Grundlage der Summenoperation kann man nun die Teilbeziehung definieren: Ein Objekt $\mathrm{x}$ soll genau dann als Teil eines Objekts y gelten. notiert als $\mathrm{x}$ cy. wenn die Zusammenfassung von $\mathrm{x}$ und $\mathrm{y}$ gleich $\mathrm{y}$ ist - in diesem Fall liegt $\mathrm{x}$ bereits in $\mathrm{y}$. Das heißt. wir legen fest: $\mathrm{x} \underline{\mathrm{c}} \mathrm{y} \leftrightarrow \mathrm{xuy}=\mathrm{y}$. Nach dieser Definition ist jedes Objekt Teil von sich selbst: um diesen Crenzfall auszuschließen. kann man die Relation des echten Teils. notiert als $x \subset y$, formulieren, und zwar als $x \subset y \leftrightarrow x \subseteq y$ und $\mathrm{x} \neq \mathrm{y}$. Nützlich ist femer der Begriff der Uberlappung zweier Entitäten $x . y$, notiert als $\mathrm{x} \circ \mathrm{y}:$ Zwei Entitäten überiappen sich genau dann. wenn sie einen gemeinsamen Teil haben: $x \circ y \leftrightarrow$ es gibt ein $z$ mit $z \subseteq x$ und $z \subseteq y$.

Eine weitere Festlegung, die wir aus formalen Gründen treffen müssen. ist die der relativen Komplementarität: Wenn eine Entität $x$ echter Teil einer anderen Entität $y$ ist, dann gibt es ein Komplement von $x$ relativ zu $y$ - oder formal: wenn $x \subset y$, dann gibt es ein $z$ mit $x\lrcorner z=y$, soda $\beta$ nicht gilt $x \circ z$. Schließlich ist es sinnvoll zu fordern, daß keine Entität Teil aller anderen Entitäten ist. d.h. daß es kein Nullelement gibt.

Verbandsstrukturen, die nicht allzu viele Elemente enthalten. können recht gut mit sogenannten Hasse-Diagrammen veranschaulicht werden. In ihnen werden Elemente durch Kreise dargestellt. und die Teilbeziehung durch Linien. wobei das größere Element über dem kleineren liegt. Ein Summenhalbverband mit drei Grundelementen x,y,z hat dann das in Abbildung (1) dargestellte Hasse-Diagramm. Charakteristisch für Hasse-Diagramme von Summenhalbverbänden ist. daß es ein Element gibt, das als Zusammenfassung aller Elemente gilt: dieses steht an der Spitze einer Art Kegel.

Es ist offensichtlich, daß bereits ein Verband mit wenig mehr Elementen zu ganz unübersichtlichen Hasse-Diagrammen führen würde. Man kann jedoch festlegen, daß die Elemente durch Punkte in einer Ebene repräsentiert werden (ähnlich wie in sogenannten Venn-Diagrammen), und daß die Teile eines Elements durch darunterliegende Punkte repräsentiert werden. Wir erhalten damit Repräsentationen der in Abbildung (2) dargesteliten Art. 


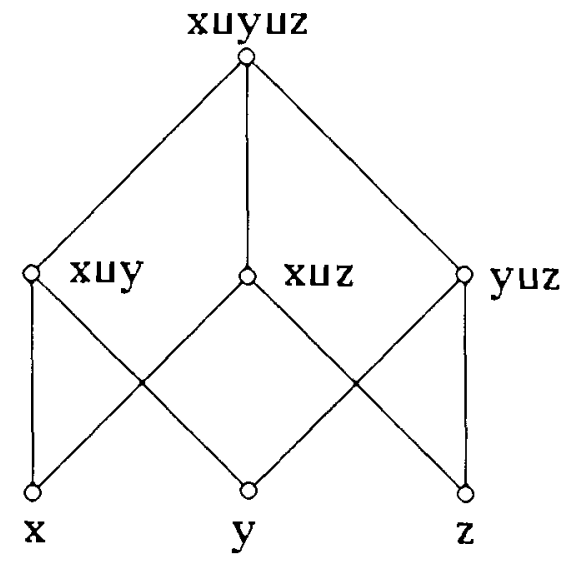

Abbildung 1: Hasse-Diagramm

eines siebenelementigen Summen-Halbverbandes ohne Nullelement

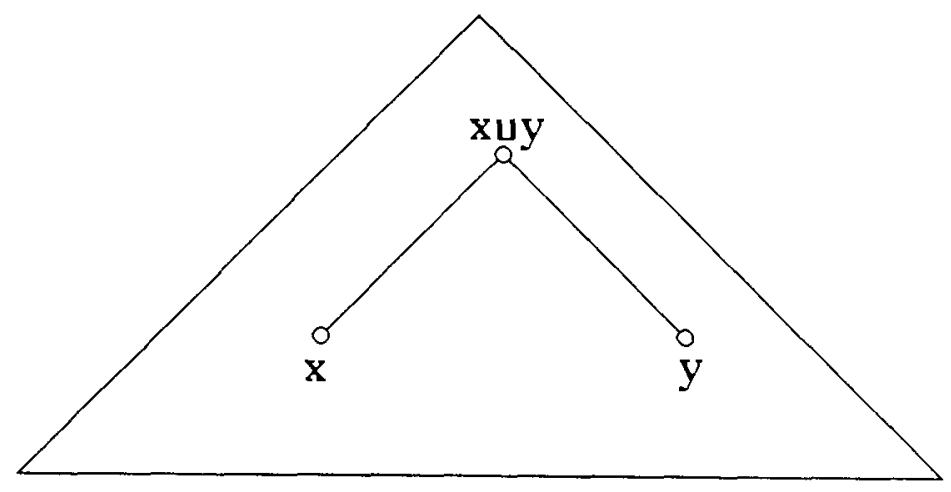

Abbildung 2: Hasse-Venn-Diagramm eines Summenhalbverbandes

Angenommen, der Bereich der Objekte, über den wir sprechen wollen, weist die Form eines Summenhalbverbandes auf. Wie können nun die zentralen Begriffe des kumulativen und des gequantelten Prädikats charakterisiert werden? Zunächst nehmen wir wie üblich an, daß die Bedeutung oder Extension eines Prädikats die Menge der Entitäten ist, auf die es zutrifft. Die Extension von kumulativen Prädikaten hat dann die Eigenschaft: Wenn immer zwei Entitäten $x, y$ in ihr 
liegen, dann liegt auch die Entität xuy in ihr. Die Extension von gequantelten Prädikaten hingegen hat die Eigenschaft: Wenn immer eine Entität $x$ in ihr liegt, dann liegt kein echter Teil $y$ von $x, y \subset x$, in ihr. (Dies hat natürlich zur Folge, daß, wenn immer zwei verschiedene $x, y$ in ihr liegen, deren Summe xuy nicht in ihr liegt).

Schließen sich Kumulativität und Gequanteltheit gegenseitig aus? Das scheint zunächst so, allerdings gibt es einen Grenzfall, wo sich beide überlappen, nämlich bei singulären Prädikaten, das sind Prädikate, die nur auf eine einzige Entität zutreffen. Ein singuläres Prädikat ist kumulativ: wenn es auf $x$ zutrifft, dann trifft es auch auf $x u x$ zu (da ja gilt: $x u x=x$ ). Es ist auch gequantelt, da es keinen echten Teil von $x$ gibt, auf den es zutreffen würde (ein echter Teil von $x$ wäre ja von $x$ verschieden). So ist es sinnvoll, den Begriff des strikt kumulativen Prädikats einzuführen. Dabei handelt es sich um nicht-singuläre Prädikate, die kumulativ sind. Es gilt, daß strikt kumulative Prädikate und gequantelte Prädikate sich ausschließen.

Ein weiterer wichtiger Begriff ist der des Atoms eines Prädikats, eines kleinsten Teilchens in der Extension eines Prädikats. $x$ ist ein Atom eines Prädikats, wenn $x$ in dessen Extension liegt und es keinen echten Teil y von $\mathrm{x}$ gibt, d.h. kein y mit ycx, soda $\beta$ auch dieser echte Teil y in der Extension des Prädikats liegt. Unter einem atomaren Prädikat sei ein Prädikat verstanden. für das gilt: Alle Entitäten, auf die es zutrifft, enthalten als Teil ein Atom relativ zu diesem Prädikat.

Wie verhält sich die Atomarität zu der Kumulativität und Gequanteltheit von Prädikaten? Es ist offensichtlich, daß jede Entität in der Extension eines gequantelten Prädikats zugleich ein Atom des Prädikats ist. Kumulative Prädikate hingegen haben nicht notwendigerweise Atome.

In Venn-Diagrammen können Extensionen bekanntlich durch Teilgebiete dargestellt werden. In Hasse-Venn-Diagrammen von Summenverbänden ergeben sich dabei charakteristische Figuren für kumulative bzw. gequantelte Extensionen, wie in Abbildung (3) veranschaulicht.

Es liegt nahe, Ausdrücke wie Apfel oder Apfelmus als Prädikate mit kumulativer Extension zu charakterisieren, und Ausdrücke wie drei Apfel oder ein Pfund Apfelmus als Prädikate mit gequantelter Extension. Allerdings sollten wir uns darüber klar werden, wie diese Ausdrücke semantisch zusammenhängen, in welcher Bedeutungsbeziehung beispielsweise Apfel zu drei Äpfel steht und wie beide zu dem offensichtlich zugrundeliegenden Individualnomen Apfel stehen. 


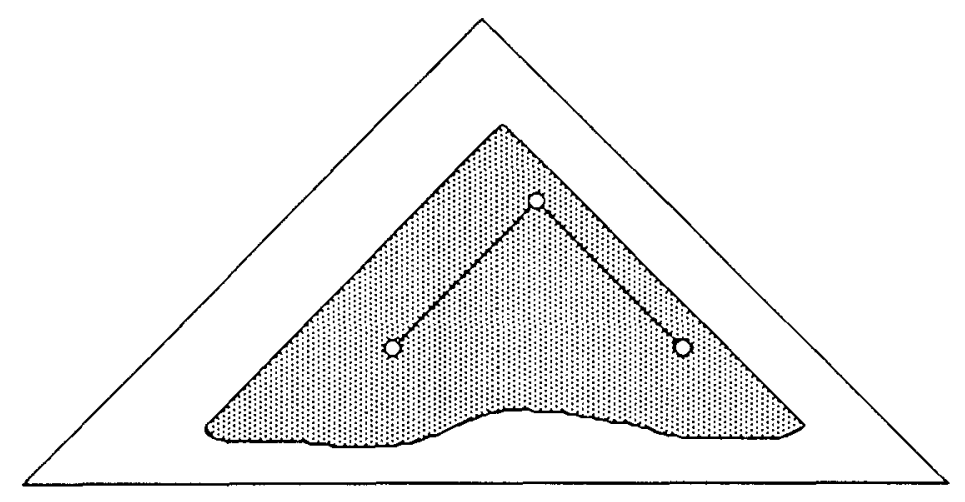

Abbildung 3a: Kumulative Extension

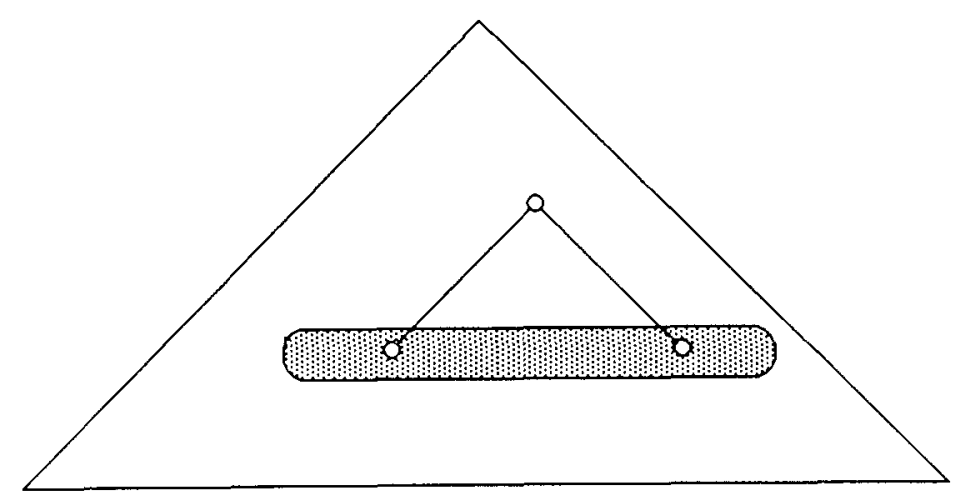

Abbildung 3b: Gequantelte Extension

Hier ist der Begriff der Maßfunktion von zentraler Bedeutung. Darunter versteht man eine Zuordnung von Entitäten zu Zahlen. Beispielsweise kann man das Temperaturmaß "Grad Celsius" als eine Zuordung von Objekten zu Zahlen verstehen: Jedem Objekt $\mathrm{x}$ wird seine Temperatur in Grad Celsius ${ }^{\circ} \mathrm{C}(\mathrm{x})$ zugeordnet, z.B. ${ }^{\circ} \mathrm{C}(\mathrm{x})=24$, falls $\mathrm{x} 24 \mathrm{Grad}$ Celsius warm ist. Ein anderes Beispiel ist das Massema $\beta$ "Pfund"; jedem Objekt $x$ wird seine Masse in Pfund $P f(x)$ zugeordnet.

Es gibt nun einen wichtigen Unterschied zwischen Maßen wie Grad Celsius (oder Karat als Goldreinheitsmaß oder dem IQ als Intelligenzmaß) auf der einen Seite und Maßen wie Pfund (oder Liter als Hohlmaß oder holländische Gulden als Wertmaß) auf der anderen Seite: erstere 
sind sogenannte intensive. letztere extensive Maße. Extensive Maße unterscheiden sich von intensiven darin. daß sie in einer systematischen Beziehung zu der Zusammenfassung der Objekte stehen. deren $\mathrm{Ma} \beta$ sie sind. Diese Beziehung wird als Additivität bezeichnet. Sie drückt beispielsweise aus: wenn $\mathrm{x} 3$ Liter Wasser sind und y 4 Liter Wasser, und wenn $x$ und $y$ sich nicht überlappen, dann sind $x$ und y zusammen 7 Liter Wasser. Oder allgemein: Wenn $m$ eine additive Maßfunktion ist und $x, y$ zwei meßbare Objekte sind. für die nicht $x \subset y$ gilt, so gilt $m(x \sqcup y)$ $=\mathrm{m}(\mathrm{x})+\mathrm{m}(\mathrm{y})$. Für intensive Maße ist dies offensichtlich nicht der Fall; wenn wir beispielsweise Wasser mit $20{ }^{\circ} \mathrm{C}$ und Wasser mit $24^{\circ} \mathrm{C}$ zusammenschütten, so erhalten wir keinesfalls Wasser mit $44{ }^{\circ} \mathrm{C}$.

Es wurde eben bereits angedeutet, daß man die Zusammenfassung von Objekten als Summenoperation $u$ deuten und damit extensive Maßfunktionen definieren kann. Durch diese Definition erzwingen wir, daß die Objekte, die relativ zu einer extensiven Maßfunktion einen festen Wert einnehmen, eine gequantelte Extension ergeben. Man kann formal beweisen:

$$
\text { Wenn } m \text { eine relativ } z u \text { der Summenoperation } u \text { extensive Maßfunktion ist und } n
$$
eine feste Zahl, dann ist $\lambda x[m(x)=n]$ ein relativ zu $u$ gequanteltes Prädikat.

Dabei ist $\lambda \times[m(x)=n]$ das Prädikat. das auf alle $x$ zutrifft. die auf der Maßfunktion $m$ den Wert $n$ einnehmen. Der Lambda-Operator $\lambda$ zeigt an, welche Variable bei der Anwendung des Ausdrucks ersetzt wird; in diesem Fall ist es die einzige Variable, $x$. Es sei etwa a ein bestimmtes Objekt: dann gilt: $\lambda x[m(x)=n](a) \leftrightarrow m(a)=n$.

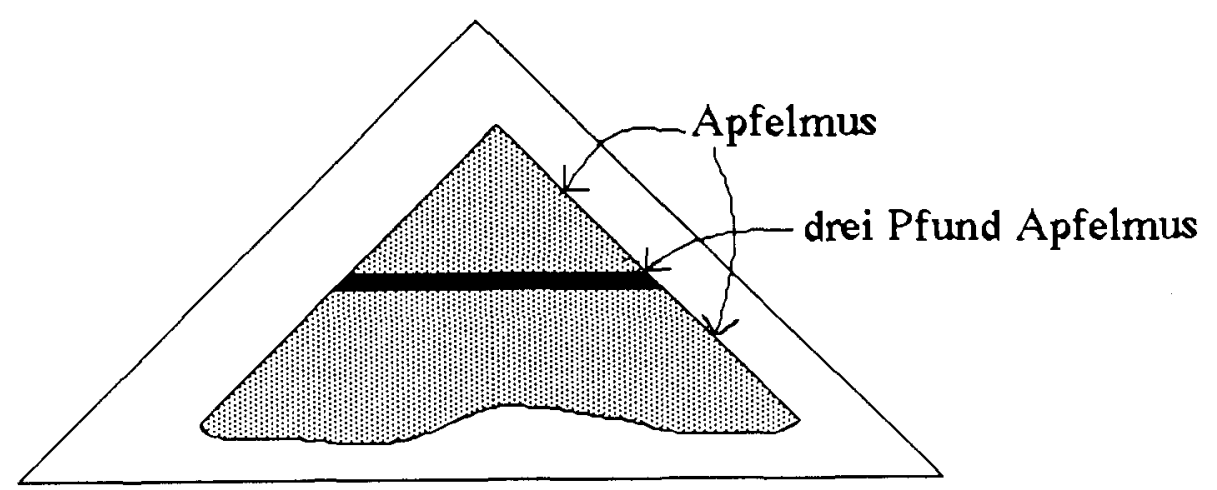

Abbildung 4: Gequantelte Teilextension

Es liegt nahe, das Numerativ in Numerativkonstruktionen, also beispielsweise Pfund in Ausdrücken wie drei Pfund Apfelmus, als extensive Maßfunktion zu interpretieren. Die Bedeutung 
von drei Pfund Apfelmus ist dann die Menge der Objekte $\mathrm{x}$, die erstens Apfelmus sind und die zweitens drei Pfund wiegen. Wenn wir annehmen, daß Apfelmus eine kumulative Extension hat, so wird durch die Anwendung des Modifikators ein Pfund eine gequantelte Teilextension spezifiziert (vgl. Abbildung 4).

In formaler Rekonstruktion handelt es sich bei Numerativen wie ein Pfund um Prädikatmodifikatoren auf der Basis von extensiven Maßfunktionen. Es sei beispielsweise Pf die Pfund zugrundeliegende Maßfunktion und Apfelmus die Interpretation von Apfelmus in unserer formalen Repräsentationssprache; dann ist die Bedeutung von drei Pfund Apfelmus wie folgt gegeben:

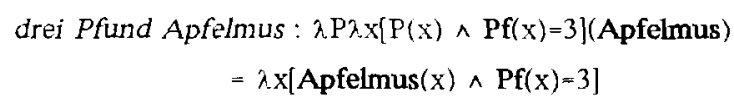

Daß der Begriff der extensiven Maßfunktion für Numerativkonstruktionen von Bedeutung ist sieht man daran, da $\beta$ Konstruktionen auf der Basis nicht-extensiver Maßfunktionen ungrammatisch sind, selbst wenn sie sonst semantisch Sinn machen würden; ein Beispiel ist * zwanzig Grad Wasser (nicht zu verwechseln mit der akzeptablen Wortbildung zwanzig-Grad-Wasser).

Man kann nun auch Ausdrücke wie ein Glas Apfelmus, drei Barren Gold, fünf Stück Zucker oder ein Strauß Blumen ähnlich interpretieren, obwohl Numerative wie Glas, Barren, Stück und Strauß sicher keine so wohldefinierten Maße wie Pfund sind. Von physikalischen Maßen wie Pfund unterscheiden sie sich beispielsweise dadurch, daß die Form von Objekten, ihr Behälter, ihr Arrangement usw. eine wesentliche Rolle spielt. Auf einer allgemeinen Darstellungsebene handelt es sich bei ihnen jedoch ebenfalls um extensive Maße. Beispielsweise sind 3 Gläser Mus und 4 Gläser Mus (die sich nicht überlappen) zusammen 7 Gläser Mus. Zwar kann man diese 7 Gläser Mus in zwei große Gläser umfüllen. Aber dies heißt lediglich nur, daß ein Maß wie Glas von der Zeit abhängig ist - dieselbe Quantität Mus kann heute auf der "Glas-Skala" den Wert 7 und morgen den Wert 2 einnehmen).

Wie verhält es sich nun mit Individualnomen-Konstruktionen wie drei Äpfel? Es liegt nahe, auch diese Konstruktionen auf extensive Maßfunktionen zurückzuführen. Erstens sind ihre Extensionen offensichtlich gequantelt. Und zweitens gibt es Fälle, in denen eine Numerativ-Konstruktion und eine Individualnomen-Konstruktion augenscheinlich dasselbe bedeuten (innerhalb einer Sprache, z.B. drei Rinder, drei Stück Vieh, sowie im Vergleich zwischen zwei Sprachen, z.B. drei Äpfel, chin. sān ge píngguð 'drei Stück Apfel'). Im Unterschied zu Numerativkonstruk-

tionen auf der Basis von Massennomina kann man jedoch annehmen, daß die Maßfunktion hier in die Bedeutung von Individualnomina "eingebaut" ist. Ein Individualnomen wie Apfel hat somit eine komplexere Bedeutung als Apfelmus : es enthält neben einer qualitativen Komponente auch 
eine quantitative, die repräsentiert wird durch eine extensive Maßfunktion. Die Pluralform in drei Apfel kann dabei als rein syntaktische Kongruenzerscheinung gedeutet werden. In der formalen Repräsentation sieht das wie folgt aus:

$$
\text { drei Äpfel : } \lambda x[\text { Apfel }(x) \wedge \mathrm{NE}(\text { Apfel })(x)=3]
$$

Hier steht NE(Apfel) für die extensive Maßfunktion "natürliche Einheit von Apfel".

Ein artikelloser Pluralausdruck wie Äpfel kann als Prädikat analysiert werden, in dem die eingebaute Maßfunktion von Apfel irgendeinen Wert (größer Null) einnehmen kann. Diese Pluralbildung ist daher ein morphologischer Prozeß, der die Anzahl-Argumentstelle des Individualnomens mit einem unspezifizierten Wert abbindet. Im folgenden sei sie zur Unterscheidung "semantischer Plural" genannt. In der formalen Repräsentation hat unser Beispiel die folgende Gestalt:

$$
\text { Apfel : } \lambda x \exists n[\operatorname{Apfel}(x) \wedge \mathrm{NE}(\text { Apfel })(x)=n]
$$

Es kann gezeigt werden, daß die Extension von Äpfel unter dieser Analyse kumulativ ist; dies entspricht unserer ursprünglichen Beobachtung.

Gegen diese Analyse liegen zwei Einwände nahe. Erstens: Ist die Unterscheidung zwischen syntaktischem Plural (Kongruenzplural) und semantischem Plural gerechtfertigt? Als Evidenz hierfür kann gelten, daß der Kongruenzplural und - singular nur mittelbar mit Mehr-oder Einzahligkeit verknüpft ist. Beispielsweise lösen Dezimalbruchzahlen immer Pluralkongruenz aus (vgl. eins komma null Äpfel/*Apfel), und bei komplexen Numeralia ist die Numeruskongruenz von der letzten Konstituente abhängig (vgl. tausendundeine $\left.N a c h t /{ }^{*} N a ̈ c h t e\right)$. Zudem gibt es viele Sprachen mit morphologischer Singular/Plural--Unterscheidung, die bei Vorhandensein eines Numerales stets den Singular einsetzen (z.B. Türkisch ü̧ elma 'drei Apfel.SG' vs. *üç elmalar 'drei Apfel.PL'); dies kann als Fehlen des Kongruenznumerus gedeutet werden.

Zweitens: Ist es gerechtfertigt, für den semantischen Plural einen unspezifizierten Wert der Anzahl-Argumentstelle anzunehmen? Ublicherweise wird man annehmen, daß ein Prädikat wie Apfel nicht auf eine beliebige Anzahl von Äpfeln zutrifft, sondern daß es schon mindestens zwei Äpfel sein müssen. Es gibt allerdings Gründe, dies anzuzweifeln. So wird man auf die Frage Hast du heute Apfel gegessen? mit ja antworten müssen, auch wenn man nur einen Apfel oder gar nur einen halben gegessen hat. Die Bedeutung von $A p f e l$ muß also diese Fälle mit umfassen. Daß ein kooperativer Sprecher das singularische Individualnomen einen Apfel verwendet, wenn er Hinweise für die Einzahl hat, und der Hörer daher häufig aus der Verwendung der Pluralform auf die Mehrzahl schliessen kann, ist nicht eigentlich Teil der Bedeutung dieser Ausdrücke, sondern kann aus allgemeinen pragmatischen Prinzipien abgeleitet werden (vgl. Grice 1975). 
236

\section{Zeitkonstitution}

Wenden wir uns nun der Bedeutung der verbalen Prädikate zu, soweit sie für unsere Grundbeispiele ausschlaggebend ist. Bekanntlich ist die grundlegende Distinktion hier die zwischen telischen und atelischen verbalen Prädikaten (oder "accomplishments" und "activities", in der Terminologie von Vendler 1957), die hier nach François (1985) als Zeitkonstitution bezeichnet sei. Informell kann diese Distinktion mithilfe des Begriffs des natürlichen Endpunkts charakterisiert werden (vgl. Garey 1957, Andersson 1972. Dahl 1981. Verkuvl 1988): Ein Verbalausdruck ist atelisch wenn er keinen "natürlichen" Endpunkt impliziert. Beispiele sind gehen und Äpfel esser. Aktivitäten dieser Art können beliebig fortgesetzt werden. Ein Verbalausdruck ist hingegen telisch. wenn er einen solchen Endpunkt impliziert. Beispiele sind fünf Kilometer gehen und drei Apfel esser, diese Aktivitäten können nicht beliebig fortgesetzt werden. sondern sind an einem bestimmten Punkt zu Ende und können dann allenfalls neu aufgenommen werden. Eine ganze Reihe von Tests ist sensitiv für diese Distinktion - die umfangreichste Zusammenstellung findet sich in Dowty (1979) - , unter anderem auch die Kombinierbarkeit mit durativen Adverbialen wie zehn Minuten lang und mit Zeitspannen-Adverbialen wie in zehn Minuten (vgl. die Grundbeispiele 1.2 ).

Wie können wir nun erklären, daß die Nominalreferenz von Verbargumenten einen Einfluß auf die Zeitkonstitution von komplexen Verbausdrücken hat? Fine angemessene Lösung dieses Problems muß davon ausgehen, daß zwischen telischen Ausdrücken und gequantelten nominalen Prädikaten einerseits und zwischen atelischen Ausdrücken und kumulativen Prädikaten andererseits gewisse Ähnlichkeiten bestehen. So wie beispielsweise ein echter Teil von drei Apfeln nicht mehr unter drei Apfel fällt, so fällt ein echter Teil eines Ereignisses von drei Kilometer laufen nicht mehr unter drei Kilometer laufen. Und so wie zwei Objekte, die unter Apfel fallen, zusammen wieder unter Äpfel fallen. so fallen zwei Laufens-Ereignisse wieder unter laufen. Daher liegt vine Rekonstruktion telischer und atelischer Verbausdrücke als gequantelte bzw. kumulative Prädikate über Ereignisse nahe, worin die Ähnlichkeit mit Nominalreferenz-Distinktionen unmittelbar deutlich wird.

Offensichtlich erklären wir dabei die Zeitkonstitutions-Unterschiede anders als durch das Vorhandensein oder Fehlen eines festen Endpunktes. Allerdings sind die beiden Sichtweisen wiederum nicht so verschieden, wie sie zunächst scheinen mögen. Denn der Begriff des festen Endpunktes läßt sich mithilfe der Begriffe der gequantelten oder kumulativen Ereignisprädikate rekonstruieren. Zunächst ist es wichtig zu sehen, da $\beta$ der Begriff des festen oder nicht-festen Endpunktes gar nicht sinnvoll auf ein einzelnes Ereignis angewendet werden hann: Wenn wir ein bestimmtes Ereignis, zum Beispiel ein bestimmtes Lauf-Ereignis, betrachten. dann hat dieses 
natürlich immer einen festen Endpunkt (ebenso wie einen festen Anfangspunkt). Es macht erst Sinn. von festen und nicht-festen Endpunkten (und Anfangspunkten) zu sprechen, wenn wir berücksichtigen, wie ein Ereignis beschrieben ist. Wenn ein Ereignis e mit laufen beschrieben wird, so ergibt sich der atelische Charakter daraus, daß es Ereignisse e' gibt, die länger dauern. $e$ als Teil enthalten und die noch immer mit laufen beschrieben werden können. Wenn dasselbe Ereignis e hingegen mit drei Kilometar laufen beschrieben wird, so ist der telische Charakter eben darauf zurückzuführen, daß es keine Ereignisse $e^{\prime}$ gibt, die länger dauern, $e^{a}$ als Teil enthalten und nit drai Kilometer laufen beschrieben werden können. Den Unterschied zwischen Telizität und Atelizität kann man also gar nicht an den Ereignissen selbst festmachen. sondern man muß hierzu auf die Fbene der Ereignisbeschreibung, oder altemativ auf die Ebene der Begriffe, hinaufsteigen.

Es können damit zwei Typen von Ereignisprädikate unterschieden werden: Erstens "Ereignisprädikate mit festem Endpunkt": dazu gehört ein Ereignisprädikat, wenn für kein Ereignis $e$ in dessen Extension gilt. daß es Teil eines Ereignisses $e^{\prime}$ in dessen Extension ist und $e^{\prime}$ länger dauert als e. Zweitens "Ereignisprädikate ohne festen Endpunkt"; dies sind die Ereignisprädikate. für die dies nicht gilt. Die Stellung dieser Distinktion zu der Distinktion kumulativ/gequantelt läßt sich nun so beschreiben, daß gequantelte Ereignisprädikate eine Teilklasse der Ereignisprädikate mit festem Endpunkt sind. und daß (strikt) kumulative Ereignisprädikate eine Teilklasse der Ereignisprädikate ohne festen Endpunkt sind (falls sie überhaupt auf Ereignisse mit unterschiedlichem Endpunkt zutreffen). ${ }^{1}$

Wenn wir die beiden Zeitkonstitutionen telisch/atelisch durch gequantelte/kumulative Prädikate über Ereignisse erfassen wollen, so kann der Einfluß der Nominalreferenz von Verbargumenten einfach als eine Art Crbertragung der Referenzweise von Verbargumenten auf den gesamten Verbausdruck erklärt werden. Beispielsweise ist drei Äpfel essen demnach gequantelt, weil drei Apfel gequantelt ist und sich diese Referenzweise auf den Gesamtausdruck drej äpfel essen überträgt.

Es mag zunächst so scheinen, als ob diese Auffassung lediglich die Theorie von Verkuyl (1972) redupliziert. Verkuyl, und später Platzack (1979), erfaßte den Einfluß von Verbargumenten mithilfe von Merkmalen wie [+Spezifizierte Quantität], die von Verbargumenten auf komplexe Verbausdrücke hochprojiziert wurden. Auf diese Weise kann man jedoch, wie Dowty (1979) argumentiert hat, die Verhältnisse in Beispielen wie (1.2) allenfalls korrekt beschreiben, nicht jedoch erklären: Nichts zwingt dazu, gerade diese und keine anderen Merkmale und Merkmalsprojektionen anzunehmen. Im Rahmen von modelltheoretischen semantischen Theorien ist es hingegen möglich. aus den angenommenen und - das ist entscheidend - unabhängig motivierten Bedeutungen der Teilausdrücke die Bedeutung komplexer Ausdrücke ohne zusätzliche Annahmen abzuleiten. Daher ist in diesem Rahmen eine explanative Lösung eher möglich. 
Es wurden verschiedene modelltheoretische Arbeiten zu dem Thema vorgelegt. Einige davon. nämlich Hoepelman (1976), Dowty (1979) und Hoepelman \& Rohrer (1980), geben die intuitive Ahnlichkeit von Distinktionen der Nominalreferenz und der Zeitkonstitution nicht wieder und wirken insgesamt recht künstlich. Andere, vor allem ter Meulen (1984), Bach (1985), Dowty (1987) und Verkuvl (1988), sind ungenügend ausgearbeitet, um die Aussagen der Theorien formal, durch logische Beweisführung, testen zu können. Der hier zu skizzierende und in Krifka (1986, 1989) formal ausgeführte Ansatz ist am ehesten mit der unabhängig entstandenen Arbeit von Hinrichs (1985) zu vergleichen. Von ihr unterscheidet er sich vor allem durch die Annahme einer wesentlich sparsameren und überschaubareren Ontologie, die beispielsweise ohne "stages" (Zeitstadien) auskommt.

Um die intuitive Ahnlichkeit von Nominalreferenz- und Zeitkonstitions-Distinktionen zu erfassen ist es nötig, die Bedeutung von Verben so anzusetzen, daß die Begriffe der kumulativen und gequantelten Referenz auf sie anwendbar sind. Dies ist möglich im Rahmen der sogenannten Ereignis-Semantik, die auf Lavidson (1967) zurückgeht und von Parsons (1980) und Carlson (1984) weiterentwickelt wurde.

In dieser semantischen Repräsentationsform sind Verben im wesentlichen Prädikate über Ereignisse. Zum Beispiel trifft laufen auf Laufens-Ereignisse und essen auf Essens-Ereignisse zu. Die Partizipanten von Ereignissen, zum Beispiel der Laufende, der Esser und das Gegessene, werden durch zweistellige Relationen mit diesen Ereignissen in Verbindung gebracht. Diese Relationen kann man mit den thematischen Rollen wie Agens und Patiens identifizieren, wie sie von ver-schiedenen linguistischen Theorien angenommen werden. Ich spreche hier und im folgenden von thematischen Relationen. ${ }^{2}$

Die intendierte Repräsentationsform wird wohl am besten anhand eines Beispiels klar. Nehmen wir das Verb essen. Semantisch ist es ein einstelliges Prädikat über Essens-Ereignisse: essen: $\lambda$ e essen(e)

Hier und im folgenden seien e, $e^{\prime}$ usw. Variablen über Ereignisse. - Das komplexe verbale Prädikat drei Äpfel essen erhält die folgende Interpretation:

$$
\text { drei Äpfel essen : } \lambda \text { e } \exists x[\text { essen }(e) \wedge \operatorname{drei-Apfel}(x) \wedge \operatorname{PAT}(\mathrm{e}, \mathrm{x})]
$$

Hier steht PAT für die thematische Relation "Patiens" und drei-Āpfel für das nominale Prädikat drei Äpfel dessen interner Aufbau hier unberücksichtigt bleiben soll. Das komplexe Prädikat trifft also auf alle Essens-Ereignisse e zu. für die es drei Apfel $x$ gibt, die in Patiens-Beziehung 
zueinander stehen. Weitere Aktanten werden auf die gleiche Weise mit der Ereignisvariable des Verbs verknüpft, zum Beispiel der Agens:

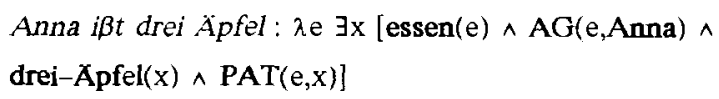

Durch einen weiteren Operator, der geeigneterweise mit dem Deklarativsatz-Modus identifiziert wird, kann dieses Prädikat in einen Satz überführt werden:

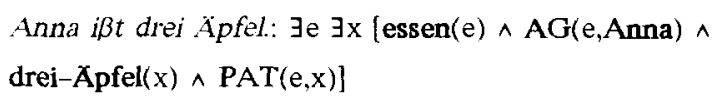

Dieser Satz ist wahr, wenn es ein Essens-Ereignis gibt, dessen Agens Anna und dessen Patiens drei Äpfel sind.

Da wir annehmen müssen, daß die hier exemplifizierten thematischen Relationen verbspezifisch sind und daher im Lexikoneintrag für Verben angegeben sein sollten, ist die in (7) angegebene Repräsentation von essen sicher ungenügend. In Krifka (1989) habe ich eine etwas weiter ausgearbeitete Larstellungsform entwickelt, die den eben gestellten Forderungen Rechnung trägt. Hier würde sie die Diskussion jedoch nur unnötig komplizieren, sodaß ich mich mit der einfacheren Darstellungsform bescheide.

Der Vorteil dieser ereignis-semantischen Repräsentationsform liegt nun darin, daß wir für Ereignisse, wie zuvor für Objekte, einen Summenverband annehmen können. Das heißt, für je zwei Ereignisse $e_{1}, e_{2}$ gibt es deren Summe $e_{1} u_{2}$; sie können in Teilbeziehung zueinander stehen, ausgedrückt durch $\mathrm{e}_{1} \check{\complement}_{2}$ bzw. e $\mathrm{e}_{1} \check{\mathrm{e}}_{2}$; sie können sich überlappen, ausgedrückt durch $e_{1} \circ_{2}$. Insbesondere kann man damit für Ereignisprädikate die Begriffe der Kumulativität und der Gequanteltheit anwenden und auf diese Weise die intuitive Ähnlichkeit von Distinktionen der Nominalreferenz und der Zeitkonstitution in der formalen Repräsentation erfassen. Beispielsweise kann man laufen als kumulatives, drei Kilometer laufen hingegen als gequanteltes EreignisPrädikat analysieren.

Die Grundidee, daß bei bestimmten thematischen Relationen die Referenzweise des Aktanten sich auf die Referenzweise des komplexen Verbausdrucks überträgt, kann nun mithilfe von einigen Annahmen über die einschlägigen thematischen Relationen formal erfaßt werden.

Es gibt eine Möglichkeit, diese Ubbertragung zu visualisieren, nämlich mithilfe von Raum/ZeitDiagrammen. In diesen Diagrammen werden Raum und Zeit durch zwei Achsen im Koordinatensystem dargestellt. Objekte können als Linien ("Weltlinien") dargestellt werden, oder besser 
als Bänder. da es auf ihre räumliche Ausdehnung in unserem Zusammenhang ankommt. Ereignisse können auf die Zeitachse. ihre Laufzeit, abgebildet werden; wenn beispielsweise ein Ereignis e ein dreistündiger Lauf ist. dann wird e einem bestimmten Zeitintervall zugeordnet.

Betrachten wir nun als Beispiel das Ereignis e. das Essen eines Glases Apfelmus (das während des Essens nach und nach verschwindet). Dieser Vorgang ist in Abbildung 5 dargestellt.

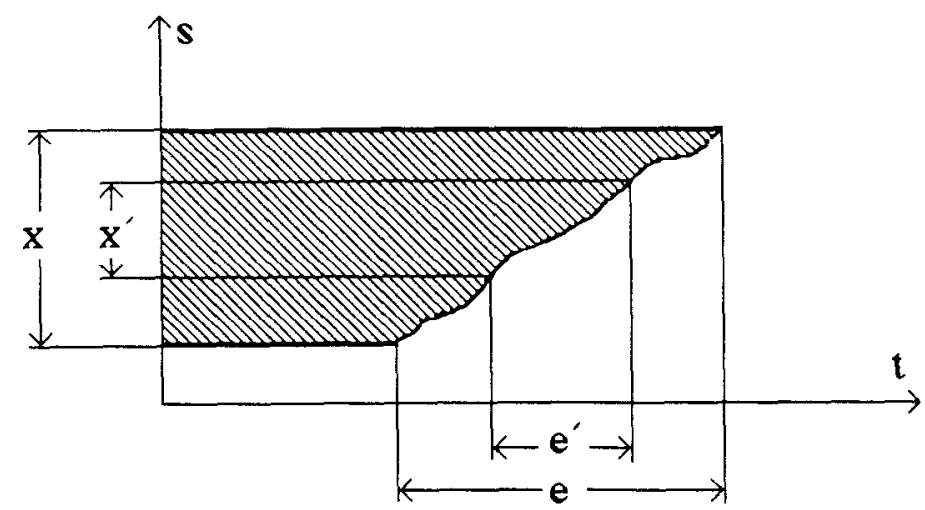

Abbildung 5: Vbertragung der Referenzweise

Durch diese idealisierte Darstellung soll die intuitive Vorstellung veranschaulicht werden, daß das Dbjekt dem Ereignis nach und nach unterworfen ist. Betrachten wir zwei mögliche Beschreibungen von $\mathrm{x}$ (und daher auch von e). Zum einen sei $\mathrm{x}$ als Apfelmus beschrieben, und daher e als Apfelmus essen. Da Apfelmus kumulativ ist, gilt normalerweise, daß dieses Prädikat auch auf echte Teile von $x$ zutrifft, zum Beispiel auf $\mathrm{x}^{\prime}$. Doch dann sollte das Prädikat Apfelmus essen ebenfalls auf Teile von e, zum Beispiel auf $e^{\prime}$, zutreffen. - Zum anderen sei $x$ als ein Glas Apfelmus beschrieben. und e daher als ein Glas Apfelmus essen. Da ein Glas Apfelmus gequantelt ist. kann kein echter Teil von $x$ mit ein Glas Apfelmus beschrieben werden. Doch dann kann auch kein echter Teil von e als ein Glas Apfelnus essen beschrieben werden.

Die Ubertragung der Referenzweise beruht, technisch gesprochen. auf einem Homomorphismus. einer struktur-erhaltenden Abbildung, von den Objekten in die Ereignisse. Dieser Homomorphismus kann mithilfe von Ubertragungs-Eigenschaften der thematischen Relationen charakterisiert werden. Um die einschlägigen Eigenschaften der thematischen Relationen zu beschreiben benötigen wir eine Reihe von Grundbegriffen. Im einzelnen haben sich dabei die folgenden Konzepta als geeignet erwiesen: 
Summativität. Eine thematische Relation $R$ sei summativ genannt, wenn aus $R(e, x)$ und $R\left(e^{\prime}, x^{\prime}\right)$ folgt: $R\left(e u e^{\prime}, x \cup x^{\prime}\right)$. Ein Beispiel: Zwei Ereignisse e, $e^{\prime}$, die jeweils als Essen eines Apfels $x$ bzw. $x^{\prime}$ beschrieben werden können, ergeben zusammengenommen ein Ereignis eve', das als das Essen zweier Äpfel xux' beschrieben werden kann. Die Summativität kann man sich als eine Erweiterung des Konzepts der Kumulativität von einstelligen Prädikaten auf zweistellige Relationen vorstellen. Es handelt sich um eine ganz allgemeine Bedingung für thematische Relationen. der beispielsweise auch die Agens-Relation unterliegt. Beispielsweise kann man aus der Existenz zweier Ereignisse, die unter drei Mädchen singen fallen, wobei sich die AgensObjekte nicht überlappen. auf die Existenz eines Summenereignisses schließen. das unter sechs Mädchen singen fällt.

Objekt-Eindeutigkeit. Wir nennen eine thematische Relation R objekt-eindeutig, wenn jedes Ereignis e zu höchstens einem Objekt $\mathrm{x}$ in R Beziehung steht. Beispielsweise steht e. das Essen eines Apfels $\mathrm{x}$, nur $\mathrm{zu} \mathrm{x}$ und $\mathrm{zu}$ nichts sonst in Patiens-Beziehung. Nicht jede thematische Beziehung ist dieser Art: beispielsweise kann das Sehen eines Hauses das Sehen eines Fensters miteinschließen.

Ereignis-Eindeutigkeit. Eine thematische Relation $\mathrm{R}$ ist ereignis-eindeutig, wenn es $\mathrm{zu}$ jedem Objekt $x$ höchstens ein Ereignis e gibt, sodaß e zu x in R-Beziehung steht. Beispielsweise kann ein Apfel $x$ nur zu einem einzigen Essens-Ereignis e Patiens sein; ein Apfeł kann nur einmal gegessen werden. Natürlich trifft dies nicht für alle thematischen Beziehungen zu; so kann beispielsweise ein und dasselbe Buch mehrmals gelesen werden.

Objekt-Abbildbarkeit. Dies beschreibt den Sachverhalt, daß es bei Bestehen einer thematischen Relation zu jedem Teil eines Ereignisses einen Teil des Objekts gibt, sodaß die Teile in derselben thematischen Relation zueinander stehen. Das heißt, wenn $R(e . x)$ und e' e gilt, so gibt es ein $x^{\prime}$ mit $x^{\prime} \subseteq x$, sodaß $R\left(e^{\prime}, x^{\prime}\right)$ gilt. Beispielsweise entspricht beim Lesen eines Buches jedem Teil des Lesens ein Teil des Buches.

Ereignis-Abbildbarkeit. Eine thematische Relation heiße ereignis-abbildbar, wenn gilt: Falls ein Ereignis und ein Objekt in der Relation zueinander stehen, so entspricht jedem Teil des objekts ein Teil des Ereignisses. sodaß diese Teile ebenfalls in der Relation zueinander stehen. Formal ausgedrückt. wenn $R(e, x)$ und $x^{\prime} \sqsubseteq x$ gilt, so gibt es ein $e^{\prime}$ mit $e^{\prime} \subseteq e$ und $R\left(e^{\prime}, x^{\prime}\right)$. Beispielsweise entspricht beim Lesen eines Buches jeder Teil des Buches einem Teil des Lesens.

Es folgen zwei Begriffe, die als Relationen zwischen Ereignisprädikaten definiert werden können:

Iterativität. Lamit wird ausgedrückt. daß ein Ereignis einer Klasse mehrfach eintritt. Es sei $P$ cin Ereignisprädikat; das iterative Ereignisprädikat $Q$ hierzu trifft auf Ereignisse zu. die sich aus mehr 
242

als einem P-Ereignis zusammensetzen. Für den hier einzig relevanten Fall endlicher Iterativität heißt dies: $\forall e\left[Q(e) \leftrightarrow \exists e_{1} . . e_{n}\left[P\left(e_{1}\right) \wedge \ldots \wedge P\left(e_{n}\right) \wedge e=e_{1} u . . u e_{n}\right]\right]$, wobei $n \geq 2$ und die $e_{1}$ paarweise verschieden sind. Beispielsweise steht (wiederholt) ein Buch lesen in iterativer Beziehung zu ein Buch lesen.

Partitivität. $P$ steht zu $Q$ in der Relation der Partitivität, wenn $P$ auf alle Teilereignisse zutriff, auf die $Q$ zutrifft. Das heißt für Ereignisprädikate $P, Q$ in Partitivitäts--Relation: $\forall \mathrm{e}[\mathrm{P}(\mathrm{e}) \leftrightarrow$ $\exists \mathrm{e}^{\prime}\left[\mathrm{Q}\left(\mathrm{e}^{\prime}\right) \wedge\right.$ ec $\left.\left.\mathrm{e}^{\prime}\right]\right]$. Ein Beispiel ist ein Buch lesen (verstanden als ein Buch von Anfang bis zum Ende lesen) und an einem Buch lesen. das dazu in Partitivitäts-Relation stellt: Teile von Ereignisse, die unter ein Buch lesen fałlen. können mit an einem Buch lesen bezeichnet werden.

Mithilfe dieser Grundbegriffe können wir nun untersuchen, welche Eigenschaften wir für die thematische Relationen anzunehmen haben, die eine Ubertragung der Referenzweise von nominaien Argumenten auf den verbalen Gesamtausdruck bewirken. Wir betrachten dabei nur den einfachsten Fall eines verbalen Ausdrucks mit einem nominalen Aktanten, der in der oben skizzierten Repräsentationsform die folgende Darstellung erhält:

$$
\text { V-GESAMT: } \lambda e \exists x[\operatorname{VERB}(e) \wedge \operatorname{NOMEN}(x) \wedge \operatorname{TH}-\operatorname{REL}(e, x)]
$$

Hier steht VERB für das verbale Prädikat, NOMEN für das nominale Prädikat, TH-REL für die thematische Relation und V-GESAMT für den verbalen Gesamtausdruck.

Eine Voraussetzung, die für alle Fälle gilt, ist dabei, daß das verbale Prädikat kumulativ ist. Damit gleichen einfache verbale Prädikate den Massennomina, und ganz allgemein kann für sprachliche Prädikate Kumulativität als der unmarkierte Fall angenommen werden, demgegenüber die Quantelung eigens gekennzeichnet werden muß.

Die erste Frage lautet: Unter welchen Bedingungen überträgt sich die Kumulativität des nominalen Prädikats auf den Gesamtausdruck? Es kann gezeigt werden, daß dies der Fall ist, wenn das nominale Prädikat kumulativ und die thematische Relation summativ ist:

Wenn NOMEN kumulativ, VERB kumulativ und TH -REL summativ ist, dann ist V-GFSAMT kumulativ.

Bei summativen thematischen Relationen wird also die Eigenschaft der Kumulativität des nominalen Prädikats auf den verbalen Gesamtausdruck übertragen. Da die Summativität eine grundlegende Eigenschaft von vermutlich allen thematischen Relationen ist, sollte diese Referenzweisen-Ubertragung unabhängig von spezifischen verbalen Prädikaten gelten. 
Das eben angeführte Resultat genügt allerdings noch nicht, um die Verträglichkeit mit durativen Adverbialen wie drei Stunden lang zu erweisen, wenn diese, wofür in Abschnitt (3) argumentiert wird, ein nicht-gequanteltes Bezugsprädikat voraussetzen. Denn Kumulativität schließt als Randfall, nämlich bei singulären Prädikaten, Gequanteltheit nicht aus. Wir müssen bei (12) daher noch fordern, daß V-GESAMT auf mindestens zwei Ereignisse zutrifft, was im allgemeinen möglich ist.

In diesem Zusammenhang ist es interessant, sich Beispiele mit definitem oder spezifischem Objekt anzusehen, wie zum Beispiel den Brief lesen. Wenn wir annehmen, daß den Brief auf genau ein $\mathrm{x}$ zutrifft, so läßt sich dieser Ausdruck als singuläres Prädikat darstellen, das heißt als Randfall eines kumulativen Prädikats. Das heißt jedoch nicht, daß der komplexe Verbausdruck den Brief lesen ein singuläres Prädikat sein muß. Dies ist auch nicht zu erwarten, da der Ausdruck den Brief drei Stunden lang lesen unter gewissen Interpretationen von den Brief lesen zulässig ist. nämlich unter der iterativen Interpretation 'den Brief immer wieder lesen' und der ereignis-partitiven Interpretation 'an dem Brief lesen'.

Wenn $\mathrm{P}$ ein beliebiges Prädikat ist und $\mathrm{Q}$ in Iterativ-Beziehung zu P steht, dann ist Q strikt kumulativ.

Dies ist unmittelbar klar: Wenn zwei Ereignisse $e_{1}$ und $e_{2}$ aus mehreren P-Ereignissen bestehen, dann besteht auch deren Summe $e_{1}$ ve 2 aus mehreren P-Ereignissen.

Wenn $P$ ein singuläres Prädikat ist und $Q$ in Partitiv-Beziehung zu P steht, und wenn die Entität, auf die $P$ zutrifft, überhaupt echte Teile hat, dann ist $Q$ strikt kumulativ.

Dies folgt aus der Abschlußeigenschaft von Teilen einer Entität: Wenn e $e_{1}$ und e Teile eines Ereignisses e sind, dann ist auch deren Summe $e_{1}$ ve 2 ein Teil von e.

Wenn wir annehmen, daß im Deutschen Iterativität und Ereignis-Partitivität nicht eigens markiert zu werden braucht, dann folgt daraus, daß ein Ausdruck wie den Brief lesen diese Interpretationen besitzt und darin strikt kumulativ ist. Damit ist er in diesen Interpretationen mit durativen Adverbialen wie drei Stunden lang kombinierbar.

Unter welchen Bedingungen, wollen wir nun fragen, vererbt sich die Singularität des nominalen Prädikats NOMEN auf den Gesamtausdruck V-GESAMT? Dies ist beispielsweise dann der Fall, wenn die partitive Interpretation ausgeschlossen wird und für die thematische Relation EreignisEindeutigkeit angenommen wird. 
(15) Wenn NOMEN singulär ist und TH-REL ereignis-eindeutig, so ist V-GESAMT singuiär (falls es überhaupt auf ein Ereignis zutrifft)

Die Ereignis-Eindeutigkeit hat zur Folge, daß die iterative Interpretation nicht mehr möglich ist. Da NOMEN nur auf ein einziges Objekt zutrifft, kann wegen Ereignis-Eindeutigkeit von THREL auch V-GESAMT nur auf ein einziges Ereignis zutreffen. Beispiele für Verben mit ereigniseindeutigen thematischen Relationen sind Verben mit effizierten oder konsumierten Objekten. wie in den Brief schreiben oder den Apfel essen - einen Brief kann man nur einmal schreiben, einen Apfel nur einmal essen.

Betrachten wir nun die Bedingungen. unter denen sich die Quantelung des nominalen Prädikats auf den Gesamtausdruck überträgt. Dies ist dann der Fall, wenn die thematische Relation objekteindeutig, ereignis-eindeutig und objekt-abbildend ist und die iterative und die ereignis-partitive Interpretation ausgeschlossen ist.

Wenn TH-REL objekt-eindeutig, ereignis-eindeutig und objekt-abbildend ist und wenn NOMEN gequantelt ist, so ist V-GESAMT gequantelt.

Ein Beispiel hierfür ist einen Brief schreiben : Zu jedem Schreibens-Ereignis gibt es genau ein Objekt, das geschrieben wird und zu jedem Objekt, das geschrieben wurde. genau ein SchreibEreignis, und schließlich entspricht jedem Teil des Schreibens ein Teil des Objekts. Somit sollte einen Brief schreiben gequantelt sein (falls der Ausdruck nicht ereignis-partitiv interpretiert wird).

Dies ist auch tatsächlich der Fall. wie die folgenden Tests zeigen:

(17) a. in einer Stunde einen Brief schreiben

b. eine Stunde lang einen Brief schreiben

Die Kombinierbarkeit mit Zeitspannen-Adverbialen (a) zeigt die Utbertragung der Quantelung von einen Brief auf einen Brief schreiben. Die Kombination mit durativen Adverbialen (b) erzwingt eine nicht-gequantelte Interpretation; diese ist nach unserer Theorie möglich, wenn einen Brief schreiben iterativ oder ereignis-partitiv interpretiert wird, und tatsächlich hat (b) eine iterative ("immer wieder einen Brief schreiben") und eine ereignis partitive I.esart ("an einem Brief schreiben").

Die Ereignis-Eindeutigkeit ist jedoch eine zu starke Forderung, um den ganz ähnlich gelagerten folgenden Fall zu erklären:

(18) a. in einer Stunde einen Brief lesen 
b. eine Stunde lang einen Brief lesen

Es läßt sich zeigen, daß die Objekt--Eindeutigkeit und die Objekt-Abbildbarkeit, die wir für das svntaktische Objekt von lesen annehmen können, nicht genügt, aus der Quantelung von NOMEN die Quantelung von V-GESAMT abzuleiten. Allerdings kann man ableiten, daß V-GESAMT (soweit es nicht ereignis-partitiv interpretiert wird) ein atomares Prädikat ist.

Wenn NOMEN gequantelt und TH-REL objekt-eindeutig und ereignis- abbildend ist. dann ist $V$. GESAMT atomar.

Dies legt die Annahme nahe, daß Zeitspannen-Adverbiale nicht fordern. daß ihre Bezugsprädikate gequantelt sind, sondern lediglich, daß sie atomar sind. In Abschnitt (3) werde ich zeigen, daß diese Annahme gerechtfertigt ist.

Wir haben nun verschiedene Eigenschaften für thematische Relationen kennengelernt. welche die Ubertragung bestimmter Referenzweise-Eigenschaften vom nominalen Prädikat auf das verbale Gesamtprädikat sichern. Die Kombination von dreien solcher Eigenschaften ist besonders interessant, nämlich von Objekt-Eindeutigkeit, Objekt-Abbildbarkeit und Ereignis-Abbildbarkeit: sie sei Gradualität genannt. Sie drückt aus. daß das Objekt dem Verb aut eine graduelle oder inkrementelle Weise unterworfen ist, das heißt, in der Art und Weise, wie es in dem Raum-ZeitDiagramm oben dargestellt wurde.

Betrachten wir nun die Objekte verschiedener Verben in der Hinsicht, welche Eigenschaften ihre thematischen Relationen erfüllen:

\begin{tabular}{|lcccl|}
\hline Beispiel & Summativ & Gradual & $\begin{array}{l}\text { Ereignis } \\
\text { eindeut. }\end{array}$ & $\begin{array}{l}\text { Thematische } \\
\text { Relation }\end{array}$ \\
\hline einen Brief schreiben & $\mathrm{X}$ & $\mathrm{X}$ & $\mathrm{X}$ & gradual effiziert \\
einen Apfel essen & $\mathrm{X}$ & $\mathrm{X}$ & $\mathrm{X}$ & gradual konsumiert \\
einen Brief lesen & $\mathrm{X}$ & $\mathrm{X}$ & - & $\begin{array}{l}\text { gradual affiziert } \\
\text { eine Katze streicheln }\end{array}$ \\
eine Katze sehen & $\mathrm{X}$ & - & - & Stimulus affiziert \\
\hline
\end{tabular}

Die Summativität der Objektrelationen ist der Grund, weshalb sich die Kumulativität des nominalen Prädikats auf den Gesamtausdruck überträgt. Als Test hierfür dient die Kombinierbarkeit mit durativen Adverbialen und die Nicht-Kombinierbarkeit mit Zeitspannen-Adverbialen (hier darf in drei Stunden natürlich nicht in futurischer Bedeutung interpretiert werden):

(20) a. drei Stunden lang Briefe schreiben 

b. drei Stunden lang Äpfel essen
c. drei Stunden lang Briefe lesen
d. drei Stunden lang Katzen streicheln
e. drei Stunden lang Katzen sehen

(21) a. *in drei Stunden Briefe schreiben

b. *in drei Stunden Apfel essen

c. *in drei Stunden Briefe lesen

d. *in drei Stunden Katzen streicheln

e. $\quad$ in drei Stunden Katzen sehen

Die Gradualität der Objektsrelation ist hingegen verantwortlich dafür, daß der Gesamtausdruck in nicht-iterativer und nicht-progressiver Interpretation die Gequanteltheit des nominalen Prädikats übernimmt. Die Ereignis-Eindeutigkeit sorgt dafür, daß die iterative Interpretation ausgeschlossen ist. Als Test hierfür dient die Kombination mit durativen Adverbialen:
(22) a. drei Stunden lang fünf Briefe schreiben (nur partitiv)
b. drei Stunden lang fünf Äpfel essen (nur partitiv)
c. drei Stunden lang fünf Briefe lesen (nur partitiv oder iterativ)
d. drei Stunden lang fünf Katzen streicheln
e. drei Stunden lang fünf Katzen sehen

Wenn wir abschließend die entsprechende Kombinierbarkeit mit Zeitspannen-Adverbialen testen, erhalten wir Sonderinterpretationen für die nicht-gradualen Objektsrelationen. Ein Ausdruck wie in drei Stunden fünf Katzen sehen ist nur auf Ereignisse anwendbar, in denen die fünf Katzen mehr oder weniger nacheinander, auf keinen Fall aber alle gleichzeitig gesehen werden; ein Ausdruck wie in drei Stunden fünf Äpfel essen kann hingegen auf Ereignisse angewendet werden. in denen gleichzeitig an allen fün Äpfeln gegessen wird.
(23) a. in drei Stunden fünf Briefe schreiben
b. in drei Stunden fünf Äpfel essen
c. in drei Stunden fünf Briefe lesen
d. in drei Stunden fünf Katzen streicheln (nur nacheinander)
e. in drei Stunden fünf Katzen sehen (nur nacheinander)

Es zeigt sich, daß unsere formale Klassifikation verschiedener thematischer Relationen einen Einfluß auf die Akzeptabilität von Ausdrücken sowie auf ihre Interpretation besitzt. 
Gegenstand dieses Abschnitts ist die Frage, aus welchen Gründen durative Adverbiale und Zeitspannen-Adverbiale überhaupt als Test für die Zeitkonstitution dienen können.

Betrachten wir zunächst durative Adverbiale. Sie drücken offensichtlich dieselbe semantische Funktion aus wie Numerativphrasen im nominalen Bereich:

(24) a. drei Stunden lang essen

b. drei Pfund Apfelmus

In beiden Fällen darf der Bezugsausdruck nicht bereits gequantelt sein:

(25) a. *drei Stunden lang drei Äpfel essen

b. *drei Pfund drei Gläser Apfelmus

Durative Adverbiale und Numerativphrasen schränken die Extension eines Prädikats auf Entitäten ein, die einen bestimmten Wert auf einer extensiven Maßfunktion einnehmen. Zwar schließt dies formal nicht aus, daß das Bezugsprädikat gequantelt ist. Aber die typische Anwendungsbedingung für durative Adverbiale und Numerativphrasen ist, daß sie aus einem "Kontinuum" von Entitäten, die unter das Bezugsprädikat fallen, Entitäten von bestimmter Größe spezifizieren. Das heißt, sie werden in Fällen angewendet, in denen die Extension des Bezugsprädikats aus Entitäten besteht, die zu Ketten $x_{1}, x_{2}, x_{3} \ldots$ angeordnet werden können, wobei jeweils eine Entität echter Teil der nächsten ist (d.h., ... $x_{1} \sqsubset x_{2} \sqsubset x_{3} \quad \ldots$ ). Sie spezifizieren dabei jeweils eine Entität aus einer solchen Kette. Das Bezugsprädikat kann daher nicht gequantelt sein; strikt kumulative Bezugsprädikate hingegen erfüllen diese Bedingung.

Betrachten wir nun Zeitspannen-Adverbiale. Sie drücken offensichtlich aus, daß ein Ereignis, das unter das Bezugsprädikat fällt, in einem Zeitintervall der angegebenen Länge stattgefunden hat. Beispielsweise trifft

$$
\text { in einer Stunde drei Apfel essen }
$$

auf Ereignisse des Essens dreier Äpfel zu, die innerhalb einer Stunde stattgefunden haben. Man beachte dabei, daß diese Zeit nicht ganz in Anspruch genommen werden muß - auch wenn das Essen dreier Apfel nur 50 Minuten dauert, hat man sie in einer Stunde gegessen. Aus pragmatischen Gründen wird der Sprecher jedoch ein möglichst "enges" Zeitintervall wählen, und der Hörer kann darauf vertrauen und davon ausgehen, daß die Ereignis-Zeit nicht wesentlich kürzer war. Zeitspannen-Adverbiale können als skalare Prädikate im Sinne von Horn (1972) 
angesehen werden. die stets pragmatische Implikaturen dieser Art auslösen.

Wie läßt sich die Distribution von Zeitspannen-Adverbialen beschreiben? Sie können mit gequantelten Prädikaten wie fünf Äpfel essen kombiniert werden, aber auch mit Prädikaten, die nicht gequantelt sind. wie beispielsweise mit fünf Katzen sehen in der besonderen Interpretation, in der die fünf Katzen nicht gleichzeitig gesehen wurden. Sie können hingegen nicht kombiniert werden mit kumulativen Ausdrücken wie Äpfel essen oder Katzen sehen. Das hier zugrundeliegende Kriterium kann man das der "eindeutigen und ausgedehnten Atomarität" nennen: Fünf Apfel essen besitzt, als gequanteltes Prädikat, eindeutige und ausgedehnte Atome - jedes Ereignis, das darunter fällt, ist zugleich ein Atom dieses Prädikats. Fünf Katzen sehen in der erzwungenen Lesart des sukzessiven Sehens besitzt eindeutige und ausgedehnte Atome: Wenn man nacheinander je eine Katze sieht, so hat man erst beim Anblick der fünften Katze fünf Katzen gesehen. Bei Äpfel essen oder Katzen sehen liegen jedoch andere Verhältnisse vor. Wenn, wofür wir in Abschnitt (1) argumentiert haben, Apfel auch auf Teile eines Apfels zutrifft. so hat man mit dem ersten $\mathrm{Bi} \beta$ in einen Apfel bereits Äpel gegessen, und ähnlich mit dem ersten Anblick einer Katze bereits Katzen gesehen. Es gibt in diesen Fällen also keine eindeutigen und ausgedehnten Atome.

Man kann nun die Distribution von Zeitspannen-Adverbialen aus deren Pragmatik ableiten. Zeitspannen-Adverbiale drücken aus, daß ein Ereignis in einem Zeitintervall bestimmter Länge stattgefunden hat, und aus pragmatischen Gründen wird hierzu ein möglichst kleines Intervall gewählt. Dies ist jedoch nur möglich, wenn das Bezugsprädikat eindeutige und zeitlich ausgedehnte Atome besitzt; dann kann die Laufzeit eines solchen Atoms zugrundegelegt werden. Gibt es keine solchen Atome, ist man gezwungen, immer kleinere und kleinere Intervalle zu wählen - und da die Atome nicht wohldefiniert sind. ist es im allgemeinen nicht besonders informativ, dafür Zeitintervalle anzugeben.

Diese Erklärung wird dadurch unterstützt, daß Zeitspannen-Adverbiale. die ein besonders kurzes Zeitintervall ausdrücken, manchmal mit Prädikaten wie laufen oder Apfelmus essen kombinierbar sind:

Anna hat in 0,3 Sekunden Apfelmus gegessen.

Dieser Satz kann beispielsweise als ein Bericht über einen - ziemlich merkwürdigen - Wettbewerb gelten. in dem es darauf ankommt, in möglichst kurzer Zeit etwas Apfelmus zu verschlingen. In solchen Fällen können die Atome hinreichend bestimmt und somit die Anwendungsbedingungen für Zeitspannen-Adverbiale erfüllt sein. 
In diesem und dem folgenden Abschnitt wollen wir einige Anwendungen der oben entwickelten Theorie der Referenzweisen-Ubertragung zur Erklärung sprachlicher Erscheinungen betrachten. Wir beginnen mit der Semantik des Progressivs und dessen Markierungsmöglichkeiten.

Progressive Verbausdrücke kann man in erster Näherung als ereignis-partitive Verbausdrücke analysieren (vgl. hierzu Abschnitt 2). Ein Ausdruck wie to be reading a book trifft zu auf Teile von Ereignisse, die unter das Prädikat read a book fallen. Dies entspricht der Analyse des Progressivs von Bennet \&. Partee (1972) im Rahmen einer Intervall-Semantik. Allerdings wird dadurch das sogenannte Imperfektivitäts-Paradox nicht angemessen behandelt. Darunter versteht man nach Dowty (1979) das Problem, wie die semantische Relation eines progressiven (imperfektiven) Satzes wie Mary was reading a book zu dem entsprechenden perfektiven Satz Mary read a book formal dargestellt werden kann, wenn man berücksichtigt, daß nicht jeder progressive Satz die Wahrheit des entsprechenden perfektiven Satzes impliziert (in unserem Beispiel kann Mary aufgehört haben, das Buch zu lesen, bevor sie es fertiggelesen hat). Klammert man dieses Problem aus, so ist die Semantik des Progressivs angemessen über die Teilbeziehung zu erfassen.

Meist wird der Progressiv als verbale Kategorie am Verb markiert. so beispielsweise im Englischen und Französischen. Es gibt jedoch auch Sprachen, in denen eine zumindest ähnliche grammatische Kategorie an einem nominalen Argument ausgedrückt werden kann, so in bestimmten Fällen im Deutschen mit speziellen Präpositionalobjekten und. viel systematischer, im Finnischen mit dem Partitivkasus (vgl. zum Finnischen z.B. Heinämäki 1984 und allgemein zu dieser Funktion der Objektmarkierung Moravcsik 1978):

(28) The child was eating the fish when Mary came in.

(29) L'enfant mangeait le poisson quand Marie entra.

(30) Das Kind $\mathrm{a} \beta$ an dem Fisch, als Maria hereinkam.

(31) Lapsi söi kalaa kun Maija tuli silään.

Kind $a \beta$ Fisch.PART als $M$. kam herein

Diese recht erstaunliche Variationsmöglichkeit in der Progressivmarkierung kann mithilfe der oben entwickelten Theorie der Referenzweisen-Ubertragung erklärt werden. Sie gibt die Möglichkeit, den Effekt eines nominalen Operators für die gesamte Verbkonstruktion formal darzustellen.

Die Semantik des Progressivs kann näherungsweise als Ereignis-Partitiv beschrieben werden. Die Semantik des nominalen Partitivs ist ganz ähnlich analysierbar, nämlich mithilfe eines Operators. 
der die Bedeutung eines nominalen Prädikats so verändert, daß das Resultat auf die Teile der Objekte zutrifft, auf die das Ausgangsprädikat zutrifft. Wenn beispielsweise finnisch kalan 'Fisch' (Akkusativ) auf einzelne Fische zutrifft, so trifft kalaa (Partitiv) auf Teile von Fischen zu.

Progressiv und Partitiv haben demnach eine gemeinsame Grundbedeutung. Wir setzen hierfür einen Partitiv-Operator PART an. Es sei P ein Prädikat; dann treffe PART(P) genau dann auf eine Entität $x$ zu, wenn es ein $x^{\prime}$ gibt, das $x$ enthält und auf das $P$ zutrifft, das heißt:

$$
\operatorname{PART}(P)(x) \leftrightarrow \exists x^{\prime}\left[x \subseteq x^{\prime} \wedge P\left(x^{\prime}\right)\right]
$$

Im Falle des Progressivs wird der Partitiv-Operator auf das verbale Prädikat angewendet. Ein Beispiel:

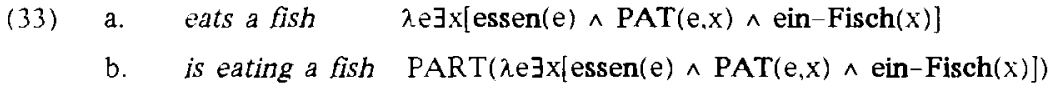

Im Falle des Partitivs wird der Partitiv auf das nominale Prädikat angewendet. Ein Beispiel:

$$
\begin{aligned}
& \text { (34) a. söi kalan } \lambda \mathrm{e} \exists \mathrm{x}[\operatorname{essen}(\mathrm{e}) \wedge \operatorname{PAT}(\mathrm{e}, \mathrm{x}) \wedge \text { ein-Fisch }(\mathrm{x})] \\
& \text { b. söi kalaa } \lambda \mathrm{e} \exists x[\operatorname{essen}(\mathrm{e}) \wedge \operatorname{PAT}(\mathrm{e}, \mathrm{x}) \wedge \operatorname{PART}(\text { ein-Fisch })(\mathrm{x})]
\end{aligned}
$$

Es läßt sich nun zeigen. da $\beta$ unter bestimmten Annahmen für die Semantik der thematischen Relation (Gradualität) das Partitiv-Beispiel aus dem Progressiv-Beispiel folgt, und unter bestimmten zusätzlichen Annahmen das Progressiv-Beispiel aus dem Partitiv-Beispiel. Das heißt, es kommt nicht darauf an, ob die Partitivität am nominalen Argument oder am verbalen Gesamtausdruck markiert wird.

Hier sind zwei abschließende Bemerkungen angebracht. Erstens: Im Finnischen dient der Partitiv auch dann zum Ausdruck des Progessivs, wenn die Bedingung der Gradualität nicht erfüllt ist (zum Beispiel bei Verben wie kaufen). Dies kann man als eine Grammatikalisierung eines ursprünglich semantisch motivierten Musters ansehen.

Zweitens: Im Deutschen wird man an-Präpositionalobjekte wohl nicht auf nominale Operatoren zurückführen wollen. Vielmehr wird man sie als alternative Kasusrahmen von Verben mit Akkusativ-Objekten behandeln. Wenn Kasusrahmen in Form einer Menge von Paaren von Kasus und zugehörigen thematischen Relationen dargestellt werden, dann kann man die hier zugrundeliegende Diathese wie folgt beschreiben:

$$
\operatorname{VERB}[\ldots,(\text { Akk, TH }), \ldots] \rightarrow \operatorname{VERB}[\ldots,(\text { an-PPO, TH-PART(TH)), ...] }
$$


Hier steht Akk für Akkusativ-Objekt und an-PPO für an-Präpositionalobjekt, TH ist eine Variable für thematische Relationen, und TH-PART sei ein Operator für thematische Relationen. der wie folgt definiert ist: Wenn TH-PART $(T H)(e, x)$, dann gibt es ein $x^{\prime}$ mit $x^{\prime} \subseteq x$ und TH(e, $\left.x^{\prime}\right)$

Das heißt, THI-PART(PAT)(e,x) heißt, daß ein Teil von $\mathrm{x}$ dem Ereignis e als Patiens unterworfen ist.

\section{Perfektivität und Definitheit}

Wir haben gesehen, daß der Einfluß einer primär nominalen Kategorie (Partitivität) auf eine verbale Kategorie (Progressivität) mithilfe der Referenzweise-CUbertragung dargestellt werden kann. Umgekehrt kann es nun auch vorkommen, daß eine primär verbale Kategorie auf eine nominale Kategorie Einfluß nimmt.

Ein gutes Beispiel hierfür finden wir in slavischen Sprachen. Bekanntlich markieren slavische Sprachen den perfektiven Aspekt, aber nicht die Definitheit der NP. Es gibt jedoch eine interessante Interaktion zwischen den beiden Kategorien, die in dem folgenden tschechischen Beispiel deutlich wird:
a. Ota pil vino.
'Ota trank Wein' (imperfektiv)
b. Ota vypil vino.
'Ota trank den Wein' (perfektiv)

Das Objekt vino wird mit imperfektiven Verben als indefinit interpretiert, mit perfektiven Verben hingegen als definit (vgl. z.B. Wierzbicka 1968 zum Polnischen, Filip 1985 zum Tschechischen).

Diese Korrelation kann mithilfe der Referenzweisen-Ubertragung erklärt werden. Denn sowohl Aspekt-Distinktionen als auch Definitheits-Distinktionen sind mit Distinktionen der Referenzweise korreliert:

Für den perfektiven Aspekt wird man annehmen, daß er die Gequanteltheit des verbalen Prädikats voraussetzt. ¿ Ublicherweise wird als Grundbedeutung des Perfektivs angenommen, daß er die Vollendung des Verbereignisses anzeigt; von einer Vollendung kann man aber nur dann sinnvoll sprechen, wenn die Ereignisse in der Extension des verbalen Prädikats nicht so beschaffen sind, $\mathrm{da} \beta$ auch echte Teile von ihnen bereits in dieser Extension liegen. Die Ereignisse müssen einen bestimmten Endpunkt haben; und wir haben bereits in Abschnitt (2) gezeigt, daß gequantelte 
Ereignis-Prädikate auf solche Ereignisse mit bestimmtem Endpunkt zutreffen. Ohne auf die Semantik des Perfektivitäts-Operators näher einzugehen (vgl. hierzu Abschnitt 6) kann man festhalten:

$\operatorname{PERF}(\mathrm{P})$ präsupponiert: $\mathrm{P}$ ist gequantelt.

Für den imperfektiven Aspekt wird man umgekehrt annehmen, daß er Gequanteltheit ausschließt: IMPERF $(\mathrm{P})$ präsupponiert: $\mathrm{P}$ ist nicht gequantelt.

Für die Definitheit wiederum wird man Singularität annehmen: Eine definite NP trifft auf eine bestimmte Entität zu, das heißt, das zugrundeliegende nominale Prädikat ist als singulär aufzufassen. Singularität impliziert aber Gequanteltheit.

Ein artikelloses Nomen im Tschechischen kann sowohl indefinit als auch definit interpretiert werden. Handelt es sich um einen Pluralausdruck oder ein Massennomen. so ist damit eine (strikt) kumulative und eine gequantelte Interpretation verbunden.

Wenn wir nun annehmen, daß Aspekt-Operatoren Skopus über den komplexen verbalen Ausdruck (d.h. über Verb und Objekt) besitzen, dann folgen unter bestimmten Annahmen für die thematische Relation des Objekts die oben angeführten Korrelationen. Die semantische Interpretation der verbalen Prädikate enthält die folgenden Formeln:
(40)
a. $\operatorname{PERF}(\lambda \mathrm{e}] x[\operatorname{VERB}(\mathrm{e}) \wedge \operatorname{NOMEN}(\mathrm{x}) \wedge \mathrm{TH}-\mathrm{REL}(\mathrm{e}, \mathrm{x})])$
b. IMPERF $(\lambda e \exists x[\operatorname{VERB}(e) \wedge \operatorname{NOMEN}(x) \wedge \operatorname{TH}-\operatorname{REL}(e, x)])$

Wir nehmen an, daß NOMEN ambig ist zwischen einer strikt kumulativen und einer singulären (d.h. gequantelten) Interpretation, und daß VERB wie üblich strikt kumulativ ist. Wir haben gesehen. daß die kumulative Interpretation von NOMEN zu einem kumulativen Gesamtausdruck führt. Dies erfüllt die Voraussetzung von PERF nicht, und somit ist diese Interpretation für (a) ausgeschlossen. Hingegen sind die Voraussetzungen für IMPERF erfüllt, und somit ist diese Interpretation für (b) möglich. Wir haben ferner gesehen, daß die gequantelte Interpretation von NOMEN zu einem gequantelten Bezugsausdruck führt, wenn TH-REL gradual und ereigniseindeutig ist. Dies erfüllt die Voraussetzungen von PERF, und somit ist diese Interpretation für (a) zulässig. Hingegen sind die Voraussetzungen für IMPERF nicht erfült, und diese Interpretation ist für (b) daher nicht zulässig. Daraus folgt, daß der perfektive Verbausdruck die definite Interpretation und der imperfektive Verbausdruck die indefinite Interpretation des Objektnomens seligiert. 
Es ist bemerkenswert, $d a \beta$ diese Behandlung der Seiteneffekte der Aspektmarkierung kompositional ist, obgleich das Phänomen selbst zunächst nicht kompositional aussieht - die Interpretation von vino hängt ja von der Aspektmarkierung ab. Die unmöglichen Interpretationen werden hier aufgrund von allgemeinen Prinzipien ausgeschlossen, ähnlich wie in die Bank ausrauben die hier unmögliche Interpretation von Bank durch die lexikalische Bedeutung von ausrauben ausgeschlossen wird.

Abschließend möchte ich auf die theoretische Bestimmung und das Verhälnis einiger grundlegender temporaler Begriffe eingehen, wie sie sich im Rahmen der hier entwickelten Auffassungen darstellen.

Unter der Zeitkonstitution ist eine Charakterisierung der inneren zeitbezogenen Eigenschaften von (komplexen) verbalen Prädikaten (oder Satzradikalen) zu verstehen. Ich habe dafür argumentiert, sie unter den allgemeineren Begriff der Referenzweise zu subsumieren, insbesondere telische Prädikate als gequantelt und atelische Prädikate als kumulativ zu verstehen. Die Zeitkonstitution ist insbesondere keine Eigenschaft einzelner lexikalischer Einträge, weil sie erst auf syntaktischer Ebene determiniert wird. Sie ist auch nicht unmittelbar an syntaktische oder morphologische Prozesse einer Sprache gekoppelt. Vielmehr ist sie als semantische Kategorie universell auf jede Sprache anzuwenden.

Unter der Aktionsart kann man, im traditionellen und von Steinitz (1981) bekräftigten Sinn. Ableitungen von (einfachen) Verben sehen. Ublicherweise werden diese Ableitungen morphologisch markiert; dies ist aber nicht unbedingt nötig. Das Besondere an der Aktionsart ist vielmehr, daß sie auf der Wortebene definiert werden kann.

Als ein Beispiel einer Aktionsart kann die in Abschnitt (4) dargestellte Ableitung von Verben mit an-Präpositionalobjekt aus Verben mit Akkusativobjekt gelten. Sie bringt zum Ausdruck, daß das Objekts-Denotat partiell dem Ereignis unterworfen wurde. Ein ähnliches Beispiel ist die Ableitung von be-Verben wie beladen aus Verben wie laden. Sie drücken umgekehrt aus, daß das Objekts-Denotat in seiner Totalität dem Ereignis unterworfen wurde. Daneben gibt es noch zahlreiche weitere Aktionsart-Typen, zum Beispiel Phasen -..Aktionsarten, die auf eine bestimmte Teilphase. z.B. den Beginn, eines Ereignisses bezugnehmen (Beispiel: los/aufen), oder IntensitätsAktionsarten, die auf eine mehr oder weniger intensive Variante der Verbereignisses bezugnimmt (z.B. lächeln aus lachen). 
Bei der Aktionsart in diesem Sinn handelt es sich klar um einen sprach-spezifischen Begriff: Eine Sprache besitzt Aktionsarten nur insofern, als in ihrer Grammatik produktive morphologische Ableitungen von Verben aus Verben vorkommen.

Für die Kategorie des Aspekts legen die Ergebnisse von Abschnitt (5) die Analysse als Markierung der Zeitkonstitution des komplexen Verbausdrucks nahe. Wir haben gesehen, daß der perfektive Aspekt die Gequanteltheit des komplexen Verbausdrucks präsupponiert (vgl. 38) und der imperfektive Aspekt die Nicht-Gequanteitheit (vgl. 39); die einfachste Annahme für die Semantik des Aspektes ist, daß die Bedeutung von Perfektiv und Imperfektiv sich darin erschöpft. Perfektiv und Imperfektiv sind keine Operatoren, die einen Ausdruck und seine Bedeutung in einen anderen und dessen Bedeutung überführen. Vielmehr überprüfen sie, ob der Verbausdruck hinsichtlich seiner Zeitkonstitution gewissen Bedingungen genügt. Die Aspektkategorien sind damit gewissermaßen Filter für semantische Interpretationen. Die Annahme dieser "mageren" Semantik für Aspektdistinktionen erscheint nicht zuletzt deshalb attraktiv, weil es in den deskriptiven Arbeiten zur Aspektsemantik nicht gelungen ist, zu einem Konsensus über die Grundbedeutung von Perfektiv und Imperfektiv zu kommen.

Von der Markierung der Zeitkonstitution unabhängig ist die Frage, wie semantische Interpretationen, die sich in der Zeitkonstitution unterscheiden, zustandekommen - weshalb beispielsweise im Deutschen ein Buch lesen telisch oder atelisch (nämlich ereignis-partitiv oder iterativ) interpretiert werden kann. Es gibt hier zwei Möglichkeiten: Erstens die Annahme von "stummen". phonologisch nicht realisierten Operatoren; und zweitens systematische Variationen in der Interpretationsmöglichkeit von lexikalischen Ausdrücken.

Um dies an einem konkreten Beispiel aufzuzeigen, betrachten wir die Iterativität. Nach der ersten Analyse besitzt das Deutsche einen stummen Iterativoperator, der ein (möglicherweise komplexes) nicht-iteratives verbales Prädikat in sein iteratives Gegenstück überführt; dieser Operator kann nach der Definition der Iterativität in Abschnitt (2) als $\lambda . P \lambda_{. e} J_{e_{1}} . e_{n}\left[P\left(e_{1}\right) \wedge . \wedge P\left(e_{n}\right)\right.$ $\left.\wedge e=e_{1} u . . u e_{n}\right]$ angegeben werden. Nach der zweiten Analyse gibt es systematische lexikalische Variationen in den lexikalischen Ausdrücken. Beispielsweise kann man annehmen, daß einstellige Verben wie husten systematisch eine semelfaktive und eine iterative Bedeutung haben. Bei Verben mit einem Patiensobjekt kann man sich vorstellen, daß die thematische Relation des Patiens entweder ereignis-eindeutig interpretiert werden kann (und damit, nach unseren Uberlegungen in Abschnitt 2, die iterative Interpretation ausschließt), oder als nicht notwendig ereignis-eindeutig und dann die iterative Interpretation zuläßt. Die zweite Analyse - die Annahme systematischer Variationen in der Interpretation lexikalischer Ausdrücke - ist restriktiver. da sie im Gegensatz zur ersten keine Skopus-Interaktion mit anderen Operatoren zuläßt.

Die klassische Analyse des Aspekts ist eire andere: Nach ihr drückt der Perfektiv aus, daß das Ereignis "von außen" betrachtet wird oder zeitlich vor der Referenzzeit liegt, während der 
Imperfektiv besagt, daß das Ereignis "von innen" betrachet wird oder die Referenzzeit in der Ereigniszeit liegt (vgl. Comrie 1976, Kamp \& Rohrer 1983). Eine Konsequenz davon ist die unterschiedliche zeitliche Relationierung von Sätzen mit telischer bzw, atelischer Zeitkonstitution: Anna trat ein. Otto ging ans Fenster. Anna duschte sich. Otto summte vor sich hin.

Die beiden Ereignisse in (41) werden als zeitlich geordnet empfunden: Zuerst trat Anna ein, dann ging Otto ans Fenster. Hingegen werden die beiden Ereignisse in (42) eher - wenn auch nicht zwingend - als zeitlich überlappend empfunden: Während Anna sich duschte, summte Otto vor sich hin.

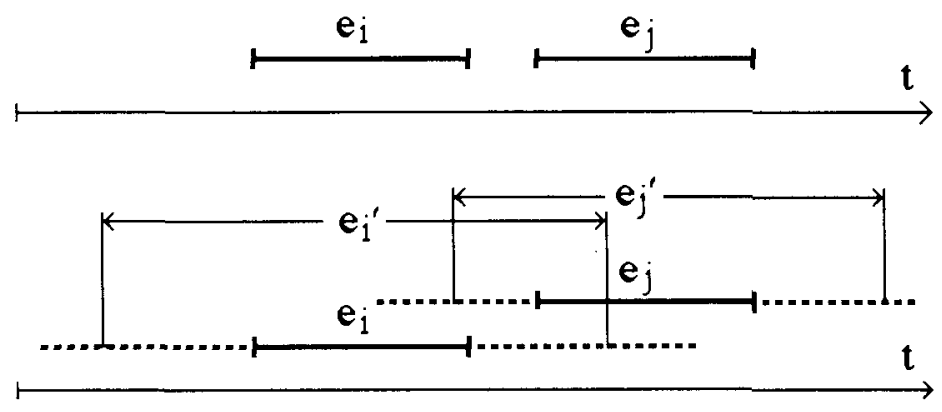

Abbildung 6: Zeitliche Ordnung von imperfektivisch und perfektivisch beschriebenen Ereignissen

Zunächst scheinen die beiden Ansichten zum Aspekt unvereinbar zu sein. Dowty (1986) hat jedoch - im Rahmen einer Intervallsemantik, aber leicht übertragbar auf eine Ereignissemantik gezeigt, daß sich die zweite Analyse aus der ersten unter Annahme einiger plausibler pragmatischer Regeln ergibt. Dowty schlägt eine allgemeinen Regel für die temporale Lokalisierung von Ereignissen vor, die im wesentlichen darauf hinausläuft: Wenn .. $S_{1}, S_{j}$.. eine Folge von Sätzen in einem Text ist, in denen keine expliziten temporalen Adverbiale vorkommen, dann liegt das Ereignis $e_{j}$, das $S_{j}$ berichtet, zeitlich nach dem Ereignis $e_{i}$, das $S_{i}$ berichtet. Wenn die Satzradikale von $S_{1}$ und $S_{1}$ beide telisch (gequantelt) sind, dann sind $e_{1}$ und $e_{1}$ auf jeden Fall zeitlich disjunkt und geordnet. Sind die Satzradikale von $S_{i}$ und $S_{j}$ atelisch, so ist dies zwar auch der Fall, es ist aber nicht ausgeschlossen. $d a \beta e_{i}$ und es Teile von größeren Ereignissen $e_{1}$ ', $e_{j}$ ' sind, die beide ebenfalls unter die Satzradikale von $S_{i}$ und $S_{j}$ fallen und sich gegenseitig zeitlich überlappen. Aus pragmatischen Gründen wird der Hörer in vielen Fällen tatsächlich annehmen, daß sich die Ereignisse zeitlich überlappen. Diese Fälle können wie in Abbildung (6) dargestellt werden. 
Hier können die verschiedenen Aspektanalysen nicht systematisch miteinander verglichen werden. Man beachte jedoch, daß für die klassische Analyse die Interaktion des Aspekts mit der Kategorie der Definitheit (vgl. Abschnitt 5) ein Rätsel bleiben muß: Es gibt einfach keinen Grund dafür, weshalb man ein Ereignis, das durch ein gequanteltes Prädikat gegeben ist, "von außen" sehen kann, ein Ereignis, das durch ein nicht-gequanteltes Prädikat gegeben ist, hingegen nicht. Die bekannten zeitlichen Ordnungseffekte - beispielsweise auch die Tatsache, daß sich Perfektivität und Präsens ausschließen - lassen sich über Dowtys ţberlegungen sinnvoll arklären. Insoweit scheint die Auffassung, der Aspekt diene zur Markierung der Zeitkonstitution komplexer Verbprädikate. der klassischen Auffassung, nach welcher er Ereignisse zur Referenzzeit in Beziehung setzt, klar überlegen.

Ich bin vielen Kollegen für die Klärung der hier vorgestellten Uberlegungen zu Dank verpflichtet, vor allem Johan van Benthem. David Dowty, Hana Filip, Franz Guenthner, Godehard Link, Barbara Partee, Theo Vennemann und Henk Zeevat.

1 Etwas genauer: Gequantelte Ereignisprädikate haben einen festen Endpunkt - es gilt ja, daß Ereignisse in ihrer Extension gar keinen echten Teile haben, die wiederum in ihrer Extension liegen. Es gibt aber ohne Zweifel nicht-gequantelte Ereignisprädikate mit festem Endpunkt - zum Beispiel das Prädikat, in dessen Extension alle Laufereignisse liegen, die um punkt 12 Uhr enden. Strikt kumulative Ereignisprädikate haben keinen festen Endpunkt, wenn sie auf mindestens zwei Ereignisse $e, e^{\prime}$ mit unterschiedlichem Endpunkt zutreffen. Denn dann treffen sie auch auf die Summe eue' zu, und damit ist e (oder e') ein echter Teil von eue'. dessen Endpunkt sich von eve' unterscheidet.

2 Für den hier vertretenen Ansatz ist die Annahme thematischer Relationen allerdings nicht zwingend. Statt ihrer kann man auch Klassen von Verben (z.B. die Klasse der Verben mit effizierten Objekten) annehmen und die Gesetzmäßigkeiten über solche Klassen formulieren. 


\section{Bibliographie}

Andersson. Sven Gunnar (1972): Aktionalität im Deutschen. Band 1. Uppsala.

Bach, Emmon (1986): "The Algebra of Events". Linguistics and Philosophy 9, 5-16.

Bäuerle, Rainer. Christoph Schwarze \& Arnim v.Stechow (1983) (eds.): Meaning, Use and the Interpretation of Language. Berlin. New York: de Gruyter.

Bennett, Michael, \& Barbara Partee (1972): Toward the Logic of Tense and Aspect in English. Bloomington: Indiana University Linguistics Club.

Carlson, Gregory N. (1984): "Thematic Roles and their Role in Semantic Interpretation". Linguistics 22, 259-279.

Comrie. Bernard (1976): Aspect. An Introduction to the Study of Verbal Aspect and Related problems. Cambridge: Cambridge University Press.

Dahl, Osten (1981): "On the Definition of the Telic/Atelic (Bounded/Non-Bounded) distinction". In Tedeschi. Ph. \& Zaenen, A. (ed.): Syntax and Semantics 14: Tense and Aspect. New York: Academic Press. 79-90.

Davidson, Donald (1967): "The Logical Form of Action Sentences". In Rescher, N. (ed.) The Logic of Decision and Action. Pittsburgh, Pittsburgh University Press. 81-95.

Dowty, David R. (1979): Word Meaning and Montague Granmar. The Semantics of Verbs and Times in Generative Semantics and in Montague's PTQ. Dordrecht: Reidel

Dowty, David R. (1986): "The Effects of Aspectual Class on the Temporal Structure of Discourse: Semantics or Pragmatics?". Linguistics and Philosophy 9, 37-62.

Dowty, David R. (1987): "Events, Aspect and NP Semantics". Paper presented at the 1987 Linguistic Institute, Stanford.

Filip, Hana (1985): Der Verbalaspekt und die Aktionsarten, dargelegt am Beispiel des Tschechischen. Magisterarbeit der Universität München.

François, Jean (1985): "Aktionsart, Aspekt und Zeitkonstitution". In Chr. Schwarze \& D. Wunderlich (eds.) (1985): Handbuch der Lexikologie. Kronberg: Atheneum. 229-249.

Garey, Howard B. (1957): "Verbal Aspects in French". Language 33, 91-110.

Grice, Paul (1975): "Logic and Conversation". In P. Cole \& J.L. Morgan (eds.) Syntax and Semantics 3: Speech Acts. New York: Academic Press. 41-58.

Heinämäki, Orvokki (1984): "Aspect in Finnish". In C. de Groot \& H. Tommola (eds.), Aspect Bound. A Vovage into the Realm of Germanic, Slavonic and Finno-Ugrian Aspectology. Dordrecht: Foris. 153-178.

Hinrichs, Erhard (1985): A Compositional Semantics for Aktionsarten and NP Reference in English. Ph.D.-Dissertation. Ohio State University.

Hoepelman. Jakob (1976): "Mass Nouns and Aspects, or: Why We Can't Eat Gingercake in an Hour". Amsterdam Papers in Formal Grammar 1.

Hoepelman. Jakob. \& Christian Rohrer (1980): "On the Mass-Count- Distinction and the French Imparfait and Passé Simple". In Chr. Rohrer (ed.) (1980): Time. Tense, and Quantifiers. Tübingen: Nieneyer. 85-112. 
Horn, Lawrence (1972): On the Semantic Properties of the Logical Operators in English. Indiana University Linguistics Club

Kamp, Hans \& Christian Rohrer (1983): "Tense in Texts". In R. Bäuerle e.a. (eds.), 250-269.

Krifka, Manfred (1986): Nominalreferenz und Zeitkonstitution. Zur Semantik von Massentermen, Pluraltermen und Aspektklassen. Dissertation, Universität München. Erscheint 1989 in der Reihe Studien zur Theoretischen Linguistik im Verlag Wilhelm Fink, München.

Krifka, Manfred (1987): "Nominal Reference and Temporal Constitution: Towards a Semantics of Quantity". Forschungsbericht der Forschungsstelle für natürlich-sprachliche Systeme der Universität Tübingen. Auch in J. Groenendijk, M. Stokhof \& F. Veltman (eds.). Proceedings of the Sixth Amsterdam Colloquium. ITLI. University of Amsterdam.

Krifka, Manfred (1989): "Nominal Reference, Temporal Constitution and Quantification in Event Semantics". Erscheint in Bartsch, R., Van Benthem, J. und P. van Emde Boas (eds.), Semantics and Contextual Expressions. Dordrecht: Foris.

Link, Godehard (1983): "The Logical Analysis of Plurals and Mass Terms: A Lattice-Theoretical Approach". In R. Bäuerle e.a. (eds.), 303-323

Moravesik, Edith (1978): "On the Case Marking of Objects". In J.H. Greenberg (ed.), Universals of Human Language Vol. 4, Stanford University Press. 249-289.

Parsons. Terence (1980): "Modifiers and Quantifiers in Natural Language". Canadian Journal of Philosophy, Supplementary Volume VI, 29-60.

Platzack, Christer (1979): The Semantic Interpretation of Aspect and Aktionsarten. A Study of Internal Time Reference in Swedish. Dordrecht: Foris.

Quine, Willard V.O. (1960): Word and Object. Cambridge, Mass.: MIT Press.

Steinitz, Renate (1981): Der Status der Kategorie "Aktionsart" in der Grammatik (oder: Gibt es Aktionsarten im Deutschen?). Linguistische Studien, Reihe A: Arbeitsberichte. Berlin: Akademie der Wissenschaften, Zentralinstitut für Sprachwissenschaft.

ter Meulen, Alice (1984): "Events, Quantities, and Individuals". In F. Landman \& F. Veltman (eds.), Varieties of Formal Semantics. Dordrecht. Foris. 259-279.

Vendler, Zeno (1962): "Each and Every, Any and All". Mind LXXI, 145-160. Auch in Z. Vendler (1967): Linguistics in Philosophy, Ithaca: Comell University Press, 70-96.

Verkuyl, Henk J. (1972): On the Compositional Nature of the Aspects. Foundations of Language Suppl. Series 15. Dordrecht: Reidel.

Verkuyl, Henk J. (1988): "Aspectual Asymmetry and Quantification". In Ehrich, V. \& Vater. H. (eds.), Temporalsemantik. Beiträge zur Linguistik der Zeitreferenz. Tübingen: Max Niemever. 220-259.

Wierzbicka, Anna (1968): "On the Semantics of the Verbal Aspect in Polish". In To Honor Roman Jakobson Essays on the occasion of his seventieth birthday. The Hague: Mouton, $2231-2249$. 